Trend nicht festsetzen. Die transatlantische Partnerschaft ist nicht etwas, das auf dem Niveau eines Allerweltsarbeitsverhältnisses dümpeln kann. Leidenschaftliches Bestreben muss es sein, den jungen Generationen ein realistisches, ausgewogenes und optimistisches Bild von Amerika zu zeigen. Dazu gehören sicherlich auch die Schattenseiten Amerikas, aber eben nicht nur. Gleiches gilt umgekehrt auch für die USA. So hat Willy Brandt in seiner Rede vor der Harvard-Universität am 5. Juni 1972 gesagt: »Sie, die Jüngeren dürfen nicht vergessen, dass die Interdependenz, die John F. Kennedy für die Staaten diesseits und jenseits des Atlantiks proklamiert hat, eine moralische, eine kulturelle, eine wirtschaftliche und politische Realität bleiben muss. "Diese Aufgabe ist in den letzten Jahren auch deshalb um so wichtiger geworden, als immer mehr in Deutschland stationierte Amerikaner abgezogen sind und noch abziehen werden, die hervorragende Botschafter und Mittler für beide Länder waren.

\section{Fazit}

Zur Jahrtausendwende schrieb das britische Magazin The Economist: »The chief question at the 1990s' end is whether Europe and America will remain a foreign-policy partnership or ignore the 20th century's lesson book and go their separate ways. "Einmal mehr bewies es seine Weitsicht. Die genannten fünf Punkte halten sich an die Lehre des 20. Jahrhunderts. Sie mögen sehr selbstverständlich klingen teilweise finden sie sich in einer kaum beachteten EUErklärung zu den transatlantischen Beziehungen vom 13. Dezember 2003 wieder - und dennoch wurden und werden sie nur unzureichend beherzigt. Dies muss sich ändern. Es ist in unserem fundamentalen Interesse, dass die transatlantische Partnerschaft fortgesetzt und in diesem Sinne die Frage des Economist beantwortet wird.

\title{
Die Krise der westlichen Allianz und die Krise des Multilateralismus
}

\author{
Joachim Krause*
}

\begin{abstract}
Since the Iraqi crisis from 2002/2003 the Atlantic Alliance has been put into question. Various authors argue that the Western Alliance has lost its substance and is becoming an empty shell. Their main argument is that the current crisis has been caused by US unilateralism, which was said to be the direct consequence of conservative and neo-conservative Republican policy strategies. This article is arguing that the causes for the transatlantic problems are considerably deeper and are preceding the Bush-Administration. These problems surely have been aggravated by the diplomacy of the BushAdministration, but they won't go away if the next election would bring about a Democratic Administration. Much of the criticism within the scholarly debate is proceeding from a simplified theoretical approach that tends to equate multilateralism with international order. It is argued in this article that one rather has to look at different expectations regarding international order on both sides of the Atlantic. While in the US multilateralism usually has been judged from its merit in terms of problem solving, Europeans have tended to value rather the procedural aspects of multilateralism (i.e. the selfbinding effect). The most important thing is to overcome this crisis by looking for new and reformed ways of multilateralism that hold the promise of both solving international problems and binding the U.S. Reforming NATO in a way that it might take over a major responsibility for international security might be a good starting point for such a political strategy.
\end{abstract}

Keywords: Transatlantische Partnerschaft, Gefährdung, Multilateralismus, Prämissen wissenschaftlicher Analysen, Perspektiven, NATO

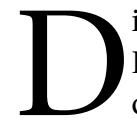
ie Irak-Krise von 2002/2003 stellt die wohl schwerste Belastung der Atlantischen Allianz seit dem Ende des Ost-West-Konflikts dar. Viele haben seither die Frage aufgeworfen, ob die Allianz überhaupt noch eine $\mathrm{Zu}$ kunft habe. Während in der Politik mittlerweile eine Sprachregelung vorherrscht, die auf größere Harmonie und Gemeinsamkeiten hindeutet, bleibt die Einschätzung der Zukunft der transatlantischen Beziehungen in der wissen-

\footnotetext{
* Prof. Dr. Joachim Krause, Direktor des Instituts für Sicherheitspolitik an der Christian-Albrechts-Universität in Kiel.
}

schaftlichen Analyse eher skeptisch. ${ }^{1}$ Viele gehen davon aus, dass die Substanz an Gemeinsamkeiten zwischen den

\footnotetext{
1 Der EU Gipfel vom 13. Dezember 2003 etwa betonte, dass die transatlantischen Beziehungen unersetzlich seien und dass sich die EU zu einer konstruktiven, ausgewogenen und zukunftsgerichteten Partnerschaft mit den USA und Kanada bekenne. Deutsche wie amerikanische Politiker betonen immer wieder die Absicht und die Notwendigkeit zusammenzuarbeiten. Dies findet sich in Dutzenden von multilateralen und bilateralen Kommuniques; vgl. Johannes Varwick, Die Zukunft der transatlantischen Sicherheitsbeziehungen, in: Österreichische Militärische Zeitschrift, 42. Jg., Heft 2, 2004, S. 141-148 ( S. 141).
} 
USA und Europa aufgebraucht sei. ${ }^{2}$ Die Irak-Krise sei der Höhepunkt einer transatlantischen Entfremdung, die in erster Linie durch einen radikalen Politikwechsel der USA verursacht worden sei. Die USA seien nicht (mehr) an einer kooperativen Weltordnung interessiert, die Europäer hingegen sowie die Überzahl der Völker und Regierungen stünden für eine multilaterale internationale Ordnung. ${ }^{3}$ Sofern es nicht einen grundsätzlichen Wandel auf Seiten der USA - oder aber einen Regimewechsel - gäbe, dürfte das Ende der transatlantischen Allianz unvermeidlich sein.

Es soll im Folgenden der Nachweis versucht werden, dass viele der oben genannten Einschätzungen auf falschen Prämissen beruhen. Insbesondere ist die Annahme eines grundsätzlichen Gegensatzes über Werte und Ziele internationaler Politik falsch. Das Hauptproblem der westlichen Politik besteht darin, dass es bei ähnlich ausgerichteten ordnungspolitischen Zielvorstellungen unterschiedliche Einschätzungen über die Nützlichkeit und Effizienz von Multilateralismus und von der Rolle militärischer Gewalt bei dem Umgang mit sicherheitspolitischen Problemen gibt. Diese Einschätzungsunterschiede haben sich vor dem Hintergrund unterschiedlicher Entwicklungen und Ereignisse während der vergangenen 15 Jahre verschärft. Sie sind keinesfalls von so grundsätzlicher Natur, dass darüber das Bündnis zerbrechen muss. Im Gegenteil, das Atlantische Bündnis könnte geradezu der Kern eines neuen Multilateralismus werden, der die Mängel des globalen Multilateralismus aufhebt.

\section{Die falschen Prämissen der meisten wissenschaftlichen Analysen}

Politische Wissenschaft sollte sich gegenüber der Politik eigentlich dadurch auszeichnen, dass sie objektiver als die Politik vorgeht und dass methodische Strenge und Abgewogenheit an Stelle emotional aufgeladener politischer Stimmung tritt. Das bedeutet in erster Linie die kritische Sichtung und Infragestellung vorherrschender Prämissen und theoretischer Vorgaben und die Beschränkung auf Behauptungen, die sich auch belegen lassen. Wenn man sich die derzeitige Debatte über die transatlantischen Beziehungen anschaut, so fällt auf, wie selten diesen Ansprüchen Genüge getan wird. Die Mehrzahl der Analysen geht von bestimmten Prämissen und theoretischen Grundannahmen aus, die in der Regel nicht in Frage gestellt werden und für die auch zumeist kein empirischer Nachweis geführt wird. Erst in der kritischen Auseinandersetzung mit diesen unhin-

2 Zu einer pessimistischen Einschätzung der Substanz der transatlantischen Beziehungen vgl. Peter van Ham, Western Unity and the Transatlantic Security Challenge, Garmisch-Partenkirchen (George C. Marshall European Center for Security Studies) 2002; siehe auch Klaus Emmerich, Atlantische Scheidung. Driften Amerika und Europa auseinander? Wien 2003.

3 Vgl. Ernst-Otto Czempiel, Weltpolitik im Umbruch. Die Pax Americana, der Terrorismus und die Zukunft der internationalen Beziehungen, München 2002; Heinrich Vogel, Das Ende des »Westens « - Tabus in den transatlantischen Beziehungen, in: Internationale Politik, Jg. 58, Heft 6 (Juni 2003), S. 27-34; Peter Rudolf, Die USA und die transatlantischen Beziehungen nach dem 11. September 2001, in: Aus Politik und Zeitgeschichte, B25 (2002), S. 7-13; Nicole Gnesotto, Übermilitarisierung amerikanischer Außenpolitik. Unilateralismus als Folge europäischer Schwäche?, in: Internationale Politik, Jg. 57, Heft 4 (April 2002), S. 43-48. terfragten und selten belegbaren Prämissen wird eine realistischere Einschätzung der transatlantischen Differenzen möglich. Die meisten kritischen Analysen lassen sich heute von drei Prämissen leiten, die bei näherem Hinsehen auf wackeligen Fundamenten stehen.

Die erste Prämisse lautet, dass die transatlantische Krise durch einen grundlegenden Wandel in der amerikanischen Außenpolitik verursacht worden ist, der durch die Aufgabe einer multilateralen internationalistischen Orientierung und die Hinwendung zu Egoismus, Unilateralismus, Militarisierung und die Missachtung internationaler Organisationen und des Völkerrechts charakterisiert sei. Dieser außenpolitische Wandel würde eine radikale Veränderung gegenüber der bisherigen Politik darstellen (mit Ausnahme der Reagan-Administration) und sei die Folge innenpolitischer Machtverschiebung weg von den Demokraten hin zu den Republikanern und innerhalb der Republikaner fort von den gemäßigten hin zu den religiösen Fundamentalisten, den Neokonservativen, den eiskalten Geschäftemachern und den Kalten Kriegern der Vergangenheit. ${ }^{4}$ Diese auch als »konservative Revolution « oder »neokonservatives Moment « bezeichnete Entwicklung sei die hauptsächliche Ursache für den Unilateralismus der USA in der Sicherheitspolitik und im Bereich des Völkerrechts und somit auch die Ursache der transatlantischen Krise. Die USA behinderten mittlerweile wirksames internationales Handeln, engagierten sich $\mathrm{zu}$ wenig in der Nord-Süd-Politik, beharrten auf eigenen Regeln und zwängen anderen ihre Regeln auf, oder sie stellten einmal gegebene Zusagen und Kompromisse in Frage. ${ }^{5}$

Auf den ersten Blick gibt es durchaus Vieles, was für diese Annahme spricht. Die undiplomatische und unilateralistische Politik der Administration von George W. Bush gerade im Bereich der Klimapolitik, die militärischen Interventionen in Afghanistan und im Irak, die befremdliche Rhetorik von der "Achse des Bösen « und nicht zuletzt die immer wieder zitierte »Bush-Doktrin « von der weltweiten militärischen Prävention scheinen für diese These $\mathrm{zu}$ sprechen. Auch Personen im engsten Umfeld von Präsident Bush, wie Vizepräsident Dick Cheney, Verteidigungsminister Donald Rumsfeld, dessen Stellvertreter Paul Wolfowitz und viele andere »knochenharte « Alt- und Neokonservative passen in dieses Bild, stammen sie doch häufig aus Kreisen, die schon während der Ära von Ronald Reagan für eine »rückwärtsgerichtete « Politik gestanden haben. ${ }^{6}$ Schaut man sich die betreffenden Argumente näher an, so werden allerdings sehr bald die Schwachstellen erkennbar.

Sollte die These von der konservativen Alleinverantwortung zutreffen, müsste ein dramatischer Wandel zumindest zur Politik der demokratischen Vorgängeradministration von Bill Clinton erkennbar werden. Dieser Nachweis ist jedoch nur schwer zu führen. Tatsächlich hatte die Clinton-

4 Vgl. Czempiel, Die amerikanische Weltordnung, in: Aus Politik und Zeitgeschichte, B 48, 2002, S. 3-6.

5 Vgl. Gert Krell, Arroganz der Macht, Arroganz der Ohnmacht, in: Aus Politik und Zeitgeschichte, B 31-32, 2003, S. 23-30 (S. 28); siehe auch Jochen Hippler, Unilateralismus der USA als Problem der internationalen Politik, ebenda, S. 15-22.

6 Vgl. John Ikenberry, The End of the Neo-Conservative Moment, in: Survival, Vol. 46, No. 1, Spring 2004, S. 7-22 (16 f.). 
Administration weitgehend all das bereits angedacht und auch schon in Konzepte umgesetzt, was heute vielen Beobachtern unangenehm auffällt. Die präemptive Anwendung militärischer Gewalt im Falle der Bedrohung durch Massenvernichtungswaffen hat auch schon die Clinton-Administration (wie die Regierungen Frankreichs) beschäftigt - nur wurde das nicht nach Außen getragen. ${ }^{7}$ Auch die IrakInvasion wurde von einer großen Mehrheit des Kongresses und der dort vertretenen Demokraten gebilligt - sie war die logische Folge einer nahezu einstimmigen Gesetzgebung des Kongresses von 1998, wonach das Problem der Rüstungskontrolle im Irak dauerhaft nur durch einen Regimewechsel in Bagdad gelöst werden könne. Die Clinton-Administration hatte 1999 und 2000 Konsultationen mit den NATOPartnern über ein militärisches Vorgehen gegen Saddam Hussein eingeleitet, allerdings ohne große Resonanz bei den Europäern zu finden, die damals davon ausgingen, dass die NATO nicht der richtige Ort dafür wäre. ${ }^{8}$

Diese vielen Gemeinsamkeiten haben auch die Kritiker der Bush-Administration registriert. Sie reagieren mit einer Hilfskonstruktion: Ihrer Argumentation zufolge waren Clinton und die Demokraten eigentlich guten Willens, mussten sich aber auf »die von der Struktur des Entscheidungsapparates vorgegebene und von den konservativen Eliten geforderte Betonung der militärischen Komponente « einlassen. ${ }^{9}$ Wieso das so sein musste, wird dann nicht weiter ausgeführt. Es verbleibt der Eindruck, es werden mehr oder weniger anonyme dunkle Kräfte (in diesem Fall »konservative Eliten «) verantwortlich gemacht. Eine wissenschaftlich befriedigende Argumentation ist das nicht. Man kann die Bush-Administration natürlich nicht von dem Vorwurf freisprechen, sie habe entscheidend dazu beigetragen, dass eine in der Wissenschaft und der Politik schon seit Jahren diskutierte Krise der transatlantischen Beziehungen ${ }^{10}$ sich dramatisch verschärft habe. Dieser Vorwurf muss aber genau so an europäische Regierungen gerichtet werden und auch gerade an Vertreter der Medien auf beiden Seiten des Atlantiks. ${ }^{11}$ Nur ist diese Feststellung einer Mitverantwortung etwas an-

\footnotetext{
7 Die Counterproliferation Initiative der Clinton-Administration legte bereits großen Wert auf Counterforce Optionen, was nichts anderes bedeutet als Angriffe auf A-B- oder C-Waffen oder entsprechende Trägersysteme, Herstellungs- bzw. Lagerungseinrichtungen. Zwar stand die Präemption Herstellungs- bzw. Lagerungseinrichtungen. Zwar stand die Präemption
nicht im Vordergrund, sie wurde aber auch nicht ausgeschlossen, insbesondere nicht bei regionalen Konflikten. Vgl. Statement by Dr. Ashton B. Carter, Assistant Secretary of Defense (International Security Policy) Before the Committee on Armed Services, US Senate, 28.4.1994; siehe dazu Joachim Krause, Die Nichtverbreitungspolitik der Clinton-Administration - Internationale Führungsmacht oder nationaler Alleingang?, in: Aus Politik und Zeitgeschichte, B 50, 1999, S. 10-17. Vgl zum französischen Konzept der Prävention (dort macht man keinen Unterschied zwischen Präemption und Prävention) Französisches Verteidigungsministerium, Programmation Militaire, 2003-2008, chapter 3, Rapport Annexé . isterium, Prog 2002 .
Paris

8 Ron Asmus, Die Herausforderung - Warum ich Präsident Bush in der Irak-Frage unterstütze, in: Frankfurter Rundschau, 20. Februar 2003 (www.fr-aktuell.de/ressorts/kultur_und_medien/feuilleton/?cnt=138688).

9 Czempiel, Weltpolitik im Umbruch, S. 81.

$10 \mathrm{Zu}$ den transatlantischen Beziehungen in den 90er Jahren vgl. David C. Gompert und F. Stephen Larrabee, America and Europe. A Partnership for a New Era. Cambridge 1997; Douglas Eden (Hrsg.), The Future of the Atlantic Community, London 1997; Phil H. Gordon (Hrsg.), NATO's Transformation. The Changing Shape of the Atlantic Alliance. Lanham 1997; David S. Yost, NATO transformed. The Alliance's new roles in in1997; David S. Yost, NATO transformed. The
ternational security. Washington, D.C. 1998.
}

11 So auch Gert Krell, Arroganz der Macht, Arroganz der Ohnmacht, a.a.O. deres als die Behauptung einer Allein- oder Hauptverantwortung der »konservativen Revolution $«$.

Vertreter einer These des grundsätzlichen konservativen Wandels der amerikanischen Außenpolitik weisen im Gegenzug darauf hin, dass die Behauptung eines revolutionären Wandels gerade von denjenigen geteilt wird, die derzeit die wackersten Verteidiger der Außenpolitik von George W. Bush sind, den Neokonservativen. Unter ihnen finden sich tatsächlich viele, die es geradezu genießen, den fundamentalen Wandel in der Außenpolitik unter George W. Bush anzupreisen. ${ }^{12}$ Allerdings ist das keinesfalls bei allen Neokonservativen der Fall. ${ }^{13}$ Aber Zitate ersetzen nicht eine saubere Politikanalyse. Diese sollte zumindest ein komplexeres Bild vom Umfeld, den Akteuren und vor allem den Anlässen für politische Entscheidungen und Richtungsgebung vermitteln. ${ }^{14}$ Sie sollte vor allem aufzeigen, wie anhand konkreter Herausforderungen unterschiedliche Akteure im bestehenden politischen System reagieren und interagieren und welche Resultate daraus abzuleiten sind. Sie muss auch Sachzwänge und objektive Entwicklungen reflektieren, auf die Politik zu reagieren hat. Politikanalyse darf sich insbesondere nicht in Vermutungen über das mehr oder weniger sinistre Treiben von Politikern bestimmter Richtungen erschöpfen, sondern sie muss aufzeigen, wie aus konkreten Situationen heraus Einzel- oder Richtungsentscheidungen fallen und welche Bedeutung dabei Idiosynkrasien gespielt haben. Die derzeitige US Administration wird deutlich von Personen geprägt, die konservativen und religiösen Vorstellungen und Ideologien anhängen. Nur waren Vertreter früherer Administrationen auch nicht frei von Ideologien, diese fielen aber zumindest in Europa nicht so negativ auf. Was am Ende im Sinne von Politikentscheidungen und Ergebnissen herauskam und herauskommt ist jedoch in einem Regierungssystem der Checks and Balances etwas völlig anderes. In der Regel sind Entscheidungsergebnisse sehr viel differenzierter $\mathrm{zu}$ beurteilen und lassen sich nicht einfach mit dem Wirken dunkler oder irrationaler Kräfte erklären.

Wie man eine differenzierte Analyse von politischen Willensbildungsprozessen in der Bush-Administration machen kann, hat der amerikanische Journalist Robert Woodward gezeigt, der in zwei Büchern den Versuch unternommen hat, die Verlaufsprozesse und Entscheidungen, die zu der Besetzung Afghanistans und Iraks geführt haben, nachzuvollziehen und Motive und Strategien herauszuarbeiten. ${ }^{15}$ In beiden Büchern kommt die derzeitige Bush-Administration auch nicht gut weg, aber es ist doch etwas ganz anderes, ein umfassendes und die Komplexität der Situationen

12 Testimony of William Kristol, Chairman, Project for the New American Century, To the Senate Foreign Relations Committee February 7, 2002.

13 Max Boot, Neocons, in: Foreign Policy, Nr. 140, Januar/Februar 2004, S. 20-28.

14 Hier sei nur hingewiesen auf das noch immer einschlägige Buch von Richard C. Snyder, H.W. Bruck and Burton Sapin, Foreign Policy Decision Making. An Approach to the Study of International Politics, New York 1962; sowie zur Policy Forschung generell Manfred G. Schmidt, Vergleichende Policy-Forschung, in: Dirk Berg-Schlosser und Ferdinand MüllerRommel (Hrsg.), Vergleichende Politikwissenschaft, s. Aufl., Opladen 1992, S. 197-212. Ansonsten liefert die seriöse amerikanische Policyforschung eine Vielzahl von Ergebnissen, die zeigen wie man vernünftig und abgewogen Politik analysiert.

15 Robert Woodward, Bush at War, New York 2002; und ders. Plan of Attack, New York u.a. 2004 
vermittelndes Bild zu bekommen, als eine Aneinanderreihung verschiedener Behauptungen, die sich häufig nur auf aus dem Zusammenhang gerissene Zitate und das Bestätigen der eigenen Auffassung durch das Zitieren anderer, gleichgesinnter Autoren stützen und deren Hauptziel es ist, die generelle Verwerflichkeit heutiger amerikanischer Außenpolitik aufzuzeigen und dies möglichst noch in eine lange Traditionsreihe von Fehlern und Missetaten der Amerikaner generell $\mathrm{zu}$ stellen. ${ }^{16}$ Eine andere Form der (Ersatz)Analyse besteht darin, die Politik der Bush-Administration (wie auch teilweise der Clinton-Administration) anhand der gerade in Mode befindlichen theoretischen Literatur zu behandeln und mit der Kritik an Theoretikern wie Huntington, Brzezinski, Kissinger, Mearsheimer und anderen gleich die ganze Politik der Bush-Administration erklären und kritisieren zu können. ${ }^{17}$ Anstelle von abgewogenen, kritischen Analysen finden sich dann schicksalsschwere Pauschalurteile.

Ein beliebtes Objekt für diese sehr oberflächliche Form der Analyse ist die Nationale Sicherheitsstrategie (NSS) der Bush-Administration vom September 2002. Die NSS wird von Kritikern in Deutschland, Frankreich und den USA gerne nur als »Bush-Doktrin « der ungehinderten Intervention und des Präventivkriegs hingestellt. Tatsächlich sind dem Thema »Präemption « (was etwas völlig anderes ist als Präventivkrieg) fünf Absätze von 36 Seiten gewidmet. Unter »Präemption « werden in dem Papier (wie in der strategischen Debatte auch) einzelne militärische Schläge verstanden, die unternommen werden, um einer ganz spezifischen Bedrohung zuvor zu kommen. Ein "präventiver Krieg « ist etwas völlig anderes: ein umfassender Einsatz militärischer Gewalt auf potentiell allen Ebenen um einen Gegner zu unterwerfen, ehe dieser stark genug ist einen selber $\mathrm{zu}$ bedrohen. Sowohl der präventive Krieg als auch die Präemption werden in der völkerrechtlichen Literatur zumeist dann als rechtmäßig angesehen, wenn eine unmittelbare Bedrohung erkennbar ist, die anderweitig nicht abzuwenden ist. ${ }^{18}$ Das Papier zur NSS macht lediglich Aussagen zu präemptiven Aktionen, nicht zum präventiven Krieg. In ihm wird die bisherige rechtliche Haltung der amerikanischen Regierung $\mathrm{zu}$ präemptiven Operationen bekräftigt, derzufolge eine unmittelbare Bedrohung vorliegen muss, ehe solche militärischen Aktionen erfolgen dürfen (was im Übrigen von den meisten Regierungen der Welt geteilt wird). Mit Blick auf neue Formen des Terrorismus und der Proliferation von Massenvernichtungswaffen wird dann allerdings festgestellt, dass die Bush-Administration die Notwendigkeit sieht, im begründeten Einzelfall auch dann präemptiv (d.h. mit Einzelaktionen gegen bestimmte Ziele) vorzugehen, wenn es noch nicht $\mathrm{zu}$ einer unmittelbaren Bedrohung gekommen ist, aber wenn damit unzumutbare Risiken verbunden

16 Eric Frey, Schwarzbuch USA, Frankfurt 2004.

17 Das ist die vorherrschende Methode bei Hajo Funke, Der amerikanische Weg. Hegemonialer Nationalismus in der US-Administration, Berlin 2003.

18 In der völkerrechtlichen Literatur wird zumeist von »anticipatory defense « gesprochen, was ein übergreifende Begriff ist, der die verschiedenen Varianten von militärischen Aktionen umgreift; vgl. Peter Malanczuk, Akehurst's Modern Introduction to International Law, seventh edition, London and New York 1997, S. 311-318. sind. ${ }^{19}$ Daraus eine Doktrin des Präventivkrieges ableiten zu wollen ist wissenschaftlich unredlich, findet aber immer wieder statt. ${ }^{20}$ Das bedeutet nicht, dass man umgekehrt die diesbezüglichen Aussagen der NSS nicht einer kritischen Würdigung unterziehen sollte, aber eine seriöse Kritik sieht anders aus. ${ }^{21}$

Wenig hilfreich sind auch andere Ansätze, die darauf hinauslaufen, die Alleinverantwortung der Bush-Administration (oder den Republikanern) für die derzeitige Krise zuzuschreiben. Die Direktorin des Forschungsinstituts der EU zu Sicherheitspolitik, Nicole Gnesotto, konstatiert in verschiedenen Schriften eine »Übermilitarisierung « der amerikanischen Außenpolitik. ${ }^{22}$ Dies macht sie, ohne zu definieren, was damit gemeint ist und wie man diese These bestätigen oder falsifizieren kann. Es fehlt völlig das Koordinatensystem, innerhalb dessen die Politik eines Landes als »übermilitarisiert «, als »normal « oder als »untermilitarisiert « bezeichnet werden kann. Dass die absoluten Militärausgaben der USA höher sind als die entsprechenden Ausgaben anderer Länder kann alleine noch kein Indikator sein, weil in den USA das Lohn- und Preisniveau höher liegt als in den meisten Staaten der Welt. Der Anteil der Militärausgaben am Bruttosozialprodukt ist nicht außergewöhnlich hoch (ca. 3\%) und im Vergleich zu anderen Akteuren (Russland, China, EU-Staaten, Indien) haben die USA weitaus weniger Soldaten unter Waffen. Auch bestehen spätestens seit dem Vietnam Krieg in den USA große Hemmnisse in Politik und Öffentlichkeit gegen den Einsatz militärischer Gewalt, insbesondere was die Inkaufnahme eigener Opfer oder von Kollateralschäden betrifft. Was die USA seit 1990 geschafft haben, ist eine relativ konsequente Modernisierung und Verkleinerung ihrer Streitkräfte. Dabei wurde versucht durch Nutzung von Informationstechnologie militärische Inter-

19 Die entsprechenden Passagen lauten wie folgt: »For centuries, international law recognized that nations need not suffer an attack before they can lawfully take action to defend themselves against forces that present an imminent danger of attack. Legal scholars and international jurists often conditioned the legitimacy of preemption on the existence of an imminent threat - most often a visible mobilization of armies, navies, and air forces preparing to attack. We must adapt the concept of imminent threat to the capabilities and objectives of today's adversaries. Rogue states and terrorists do not seek to attack us using conventional means. They know such attacks would fail. Instead, they rely on acts of terror and, potentially, the use of weapons of mass destruction - weapons that can be easily concealed, delivered covertly, and used without warning. The targets of these attacks are our military forces and our civilian population, in direct violation of one of the principal norms of the law of warfare. As was demonstrated by the losses on September 11, 2001, mass civilian casualties is the specific objective of terrorists and these losses would be exponentially more severe if terrorists acquired and used weapons of mass destruction. The United States has long maintained the option of preemptive actions to counter a sufficient threat to our national security. The greater the threat, the greater is the risk of inaction - and the more compelling the case for taking anticipatory action to defend ourselves, even if uncertainty remains as to the time and place of the enemy's attack. To forestall or prevent such hostile acts by our adversaries, the United States will, if necessary, act preemptively. The United States will not use force in all cases to preempt emerging threats, nor should nations use preemption as a pretext for aggression. Yet in an age where the enemies of civilization openly and actively seek the world's most destructive technologies, the United States cannot remain idle while dangers gather...

20 So geschehen bei E.-O. Czempiel, Die stolpernde Weltmacht, in: Aus Politik und Zeitgeschichte, B 46 (2003), S. 7-15, wo er von einer »BushDoktrin präventiver und präemptiver Kriegführung « spricht (S. 7).

21 Vgl. Robert S. Litwak, The New Calculus of Pre-emption, in: Survival, Vol 44. No. 4, Winter 2002-2003, S. 53-79; sowie Walter B. Slocombe, Force, Pre-emption and Legitimacy, in: Survival, Vol. 45, Nr. 1, Spring 2003, S. $117-130$

22 Gnesotto, Übermilitarisierung a merikanischer Außenpolitik, a.a.O. 
ventionen wieder machbar, erfolgreich und, was Opfer und Zeitaufwand betrifft, begrenzbar werden zu lassen. ${ }^{23}$ Mit den Anschlägen vom 11. September 2001 hat sich zwar insofern eine Zäsur ergeben, als dass sich in den USA die Ansicht durchgesetzt hat, dass das Land sich im Krieg mit einer politischen Kraft befinde, die der gesamten westlichen Welt aber insbesondere den USA - schweren Schaden zufügen will. Dass die USA darauf mit militärischen Mitteln reagieren, ist alleine noch keine »Militarisierung « der Außenpolitik im Sinne des mit diesem Begriff mitschwingenden Werturteils. Die USA betreiben auch auf einer Vielzahl von Feldern nicht-militärische Außenpolitik und wenden häufig mehr Ressourcen auf als ihre europäischen oder asiatischen Kritiker. Man mag mit Recht in Frage stellen, ob unter der derzeitigen Bush-Administration das optimale Gleichgewicht zwischen militärischen und nicht-militärischen Komponenten der Außenpolitik herrscht, aber auch hier muss zuvor ein klarer Maßstab für die Analyse gefunden werden.

Ein anderer Erklärungsansatz stellt darauf ab, dass die USA deshalb zum militärischen Unilateralismus neigen, weil die starke außenpolitische Stellung des US-Kongress dieses geradezu nach sich ziehen muss und weil sich die USA während des Ost-West-Konfliktes und auch danach ideologisch und institutionell zu einer Art »Sicherheitsstaat « entwickelt hätten, in dem sicherheitspolitische Themen dominierten. ${ }^{24}$

Die Behauptung, der Unilateralismus sei durch den Einfluss des Kongresses verursacht, ist plausibel. Kein Parlament der Welt hat so viel Einfluss auf die Gestaltung der Außenpolitik und keines macht auch so viel Gebrauch davon. Es stellt sich dann allerdings die Frage, warum es Zeiten geben konnte, in denen Washington vornehmlich multilateral operierte (war da der Kongress in Wartestand gegangen?) und warum es in den vergangenen 20 Jahren eine Zunahme von Unilateralismus gegeben hat? Zur Beantwortung dieser Fragen ist dann eher die Detailanalyse gefragt. So kann man für den Bereich der Sicherheitspolitik feststellen, dass der Kongress tatsächlich oftmals eine wichtige Rolle bei der Einleitung unilateraler Politik gespielt hat (zum Beispiel im Falle Bosnien-Herzegovinas und des Iraks). Aber schaut man genauer hin, so ist der Unilateralismus nicht institutionell zu erklären, sondern situationsbedingt: In allen diesen Fällen hat der US-Kongress die Administration zu einer unilateralen Politik gedrängt (bzw. diese mitgetragen), wo die überwiegende Mehrheit der Abgeordneten und Senatoren der Meinung war, dass zentrale multilaterale Institutionen (insbesondere der Sicherheitsrat der Vereinten Nationen) versagt haben. ${ }^{25}$ Ein ähnliches Bild ergibt sich mit Blick auf den Unilateralismus im wirtschaftlichen Bereich, wo Kritiker den USA (hauptsächlich dem Kongress) besonders viel Egoismus vorwerfen. Hier gäbe es zu viele Sanktionen, die

23 Vgl. Joachim Krause und Christoph Grams, Droht ein globaler Rüstungswettlauf? Perspektiven der Rüstungsindustrie im Vergleich, in: Internationale Politik, 58. Jg., Nr. 7, Juli 2003, S. 34-41.

24 Peter Rudolf, USA - sicherheitspolitische Konzeptionen und Kontroversen, in: Mir A. Ferdowsi (Hrsg.), Sicherheit und Frieden zu Beginn des 21. Jahrhunderts, München 2002, S. 149-163.

25 Vgl. Joachim Krause, Die transatlantischen Beziehungen seit dem Ende des Kalten Kriegs, in: Wichard Woyke (Hrsg.), Neue deutsche Außenpolitik, Heft 2/2003 der Zeitschrift »Politische Bildung «, S. 47-65. einseitig gegen mehr als 30 Staaten verhängt worden seien. Dieses sei ein völkerrechtlich fragwürdiges Unternehmen. ${ }^{26}$ Es gibt tatsächlich Beispiele fragwürdigen Unilateralismus und versteckten Protektionismus in der Politik des Kongresses (z.B. der Helms-Burton Act). Beides sind Sünden, die man in anderer Form auch in Europa finden kann. In vielen Fällen wurden aber Sanktionen gegen Staaten verhängt, die Bestimmungen internationaler Organisationen im Bereich der Menschenrechte oder sozialer und arbeitsrechtlicher Standards (insbesondere den Schutz gegen Kinderarbeit) verletzen oder ignorieren. Dies geschah oft auf Bitten von Nichtregierungsorganisationen, die damit den Druck auf autoritäre und rücksichtslose Regime in Afrika, Asien und Lateinamerika erhöhen wollen. Parlamente in Europa stehen teilweise unter ähnlichem Druck.

Diese Beispiele zeigen, dass man nicht einfach Unilateralismus als Untugend konstatieren und dann anhand einer Liste unilateraler Maßnahmen ein Sündenregister aufmachen kann, welches umso schwerer wiegt, je länger es wird. Viel wichtiger ist doch $\mathrm{zu}$ fragen, warum die verantwortlichen politischen Kräfte in den USA (sei es in Kongress, Administration oder Öffentlichkeit) der Meinung sind, dass bestehende Formen des Multilateralismus entweder ergänzungsbedürftig oder aber fehlerhaft sind.

Auch die Behauptung einer institutionell-ideologischen Kultur des Sicherheitsstaates ist auf den ersten Blick einleuchtend, empirisch untermauert wird sie aber auch nicht. Sie bleibt eine feuilletonistische Note, solange es kein Koordinatensystem gibt, welches es erlaubt ein Zuviel oder ein Zuwenig an Sicherheitsorientierung in der amerikanischen Außenpolitik zu bestimmen. Vor allem wird sie dem Umstand nicht gerecht, dass die USA - nicht zuletzt auch weil es so viele Staaten wollen - regionale Sicherheitsfunktionen wahrnehmen, vor denen sich andere Staaten scheuen. Dass daraus auf der einen Seite eine relative Unterschätzung, auf der anderen Seite eine relative Überschätzung der Relevanz von Sicherheitsfragen resultieren kann, ist irgendwo plausibel. Das hilft teilweise unterschiedliche Einschätzungen in Europa und den USA zu erklären, läuft aber auch in Gefahr, zum Ausgangspunkt einseitiger Schuldzuweisung zu werden. Das amerikanische Gegenstück ist dann die Behauptung des europäischen Trittbrettfahrertums.

Das alles soll keine Entschuldigung für Fehler der BushAdministration darstellen, aber die weitgehenden Pauschalvorwürfe, die sich dann leicht in generelle Vorwürfe gegen die USA umsetzen (und zur Feindbildproduktion führen), sind wissenschaftlich gesehen weitgehend nicht haltbar. Die Frage stellt sich nur, warum derartige Pauschalurteile in der wissenschaftlichen Debatte so große Konjunktur haben. Das hat vordergründig mit der aufgeheizten Atmosphäre im Zusammenhang mit der Irak-Krise und dem überaus undiplomatischen Vorgehen der Bush-Administration $\mathrm{zu}$ tun. Es gibt aber noch einen anderen Grund, der auf die zweite falsche Prämisse hinweist. Diese ist vor allem in der Wissenschaft vertreten. Sie besagt, dass das Verhalten der USA normal sei in dem Sinne, dass eine hegemoniale Macht irgend-

26 Hippler, Unilateralismus der USA, a.a.O., S. 16-17. 
wann die Tugenden des wohlmeinenden Hegemons ablege und zur uneingeschränkten Herrschaftsausübung übergehe. Anders ausgedrückt: Man ist sich so sicher in dem negativen Urteil über die USA, weil diese genau das tun, was im Sinne einer gerade vorherrschenden Theorie von ihnen erwartet wird.

Theorien hegemonialer Stabilität beherrschen seit vielen Jahren das mainstream-Denken in der IR Theoriedebatte. Der Begriff des wohlmeinenden Hegemons steht hier im Mittepunkt. Damit soll ausgedrückt werden, dass eine hegemoniale Macht (wie die USA) mit Erfolg eine kooperative internationale Ordnung aufbauen kann, wenn und solange sie sich institutionell einbindet und sich an Regeln hält, die gemeinsam mit anderen aufgestellt worden sind, wie das Völkerrecht und die Respektierung von Entscheidungen internationaler Organisationen. ${ }^{27}$ Im Prinzip sind diese Überlegungen vernünftig und logisch. Sie stellen die Anwendung von Grundprinzipien moderner kooperativer Führungsmethoden auf die internationale Politik dar. Von ihr abzugehen bedeutet in der Regel Reibungsverluste zu riskieren bzw. auch zu scheitern. ${ }^{28}$

Allerdings wird dieses plausible Prinzip zu einer dogmatischen Falle, wenn man es zu einer allgemeinen empirischen Theorie verdichtet, die Axiome aufstellt, die dann der Realität auf mehr oder weniger grobe Weise übergestülpt werden und eigentlich nur noch »binäre « Antworten erlauben - d.h. entweder die USA verhalten sich wie ein guter oder wie ein schlechter Hegemon. Ein typisches Zeichen für eine solche Tendenz zur Verengung ist die mittlerweile bei nahezu allen Anhängern dieser Theorie - sei es solche, die von einem institutionalistischen oder einem neorealistischen Hintergrund kommen - vorhandene Vorstellung, dass nur die Unterwerfung des Hegemons unter die Regeln des Multilateralismus dazu führe, dass dieser als wohlmeinend anerkannt werde. ${ }^{29}$ Die Frage stellt sich nur: Warum muss es gerade nur die Beachtung der Regeln des Multilateralismus sein? Wer hat dies festgelegt und auf welcher Logik beruht dies? Hier liegt doch eine willkürliche Festlegung vor. Es ist von Kritikern $\mathrm{zu}$ recht darauf verwiesen worden, dass im Falle der USA die Perzeption der guten Intentionen während der vergangenen Jahrzehnte auf anderen Faktoren beruht hat, die weitaus grundständiger sind (wie der demokratische Charakter des amerikanischen Systems sowie die Tradition

27 Vgl. G. John Ikenberry, After Victory. Institutions, Strategic Restraint, and the Rebuilding of Order After Major Wars. Princeton, N.J. und Oxford 2001; Michael J. Glennon, Why the Security Council Failed, in: Foreign Affairs, Jg. 82, Nr. 3, May/June 2003, S. 16-35; siehe auch Stanley Hoffmann, Gulliver's Troubles or the Setting of American Foreign Policy, New York 1968; Patrick Stewart, Multilateralism and U.S. Foreign Policy. Ambivalent Engagement, Boulder Col. 2002.

28 Vgl. Joseph Nye, Bound to Lead: The Changing Nature of American Power, New York 2001; s.a. Michael Mazaar, Acting Like a Leader, in: Survival, Vol. 44, Nr. 4, Winter 2003-2004, S. 107-120.

29 Typische Aussagen lauten wie folgt: »For multilateralism to work, rules must bind the hegemon as well as the smaller players «....»Without the self-binding of the hegemon, multilateral organisations become empty shells«; Zitate bei Richard Higgot, Multilateral Economic Institutions and the Limits to Global Governance, in: William Wallace und Young Soogil (Hrsg.), Asia and Europe. Global Governance as a Challenge to Cooperation, Tokio 2004, S. 95-120 (S. 115), das letzte Zitat ist von Lisa Martin. der USA als nicht-imperiale Macht). ${ }^{30}$ Und auch diejenigen, die den USA durchweg schlechte Absichten unterstellen und für die Washington schon immer ein böser Hegemon gewesen ist, machen ganz andere Gründe geltend als die Nichteinhaltung von Regeln internationaler Institutionen: Meistens ist es der kapitalistische Charakter, der angebliche Einfluss der Juden oder anderer Bevölkerungsgruppen auf die Politik oder einfach Hass auf die Moderne. ${ }^{31}$

Eine andere Gefahr bei dieser Theorierichtung ist die, dass historische Gesetzmäßigkeiten formuliert werden, die über Jahrtausende Gültigkeit beanspruchen sollen und die gleichzeitig zur Erklärung bzw. Prognose der aktuellen amerikanischen Politik herangezogen werden. Es gibt eine aus den USA kommende Schule historisch-strukturalistischer Analyse, die internationale Systeme unter dem Aspekt der hegemonialen Stabilität betrachtet. Im Ergebnis sind Studien herausgekommen, die versuchen nachzuweisen, dass es gewisse immer wiederkehrende Stadien im Verhalten von Großmächten gibt - insbesondere wenn diese alleiniger Hegemon sind - und die letztendlich zum Zerfall dieser Macht beitragen. Meistens beginne dieser Zerfall mit der machtpolitischen Anmaßung und Überdehnung des Imperiums. Dabei finden sich explizite Theorien des Aufstiegs und Niedergangs großer Mächte, die auch teilweise formale Modelle verwenden und nach Zyklen differenzieren. ${ }^{32}$ So interessant diese historischen Großtheorien sind, ihre Anwendung auf die aktuelle Politik sollte mit großer Skepsis betrachtet werden, denn der Übergang zum historischen Determinismus wenn nicht gar zur Metaphysik ist möglicherweise nur fließend. Beispiele aus der jüngeren Vergangenheit haben dies immer wieder gezeigt. ${ }^{33}$ Aber das Vertrauen in die Gültigkeit und unmittelbare Anwendbarkeit solcher Theorien scheint ungebrochen $\mathrm{zu}$ sein, wie das nachfolgende Zitat beweist: »Das amerikanische Selbstbewusstsein, die unangefochtene Hegemonialmacht zu sein, die Rolle des Weltpolizisten zu übernehmen und die internationale Ordnung $\mathrm{zu}$ stiften... muss es ausschließen, dass die USA eine andere weltordnungspolitische Option wie etwa das idealistische Projekt des Multilateralismus unter dem Dach der Vereinten Nationen akzeptiert. Genau dies lehrt uns die Geschichte von Aufstieg und Niedergang der großen Mächte. « ${ }^{34}$ Der hier deutlich werdende Determinismus schließt eigentlich jegliches politisches Lernen, jeden historischen Fortschritt und

30 Randall L. Schwaller, The Problem of International Order Revisited: A Review Essay, in: International Security, Jg. 26, Nr. 1 (Sommer 1999), S. 161-186; William C. Wohlforth, The Stability of a Unipolar World, in: International Security, Jg. 24, Nr. 1 (Sommer 1999), S. 5-41

31 Vgl. Dan Diner, Feindbild USA. Über die Beständigkeit eines Ressentiments, München 2002.

32 William R. Thompson, On Global War. Historical-Structural Approaches to World Politics, Columbia, S.C. 1988; William Thompson, The Emergence of the Global Political Economy, London und New York 2000; Karen A. Rasler und William R. Thompson, The Great Powers and Global Struggle, 1490-1990, Lexington: 1994; Charles Kindleberger, World Economic Primacy: 1500 to 1990, New York 1996; George Modelski, The Study of Long Cycles, in: ders. (Hrsg.), Exploring Long Cycles, Boulder, Col. 1987; Paul Kennedy, Aufstieg und Fall der großen Mächte. Ökonomischer Wandel und militärischer Konflikt von 1500 bis 2000, Frankfurt a M. 1987.

33 So galt Paul Kennedys These, wonach die USA Ende der 80er Jahre in eine Phase des Niedergangs eintreten würden, schon nach wenigen Jahren widerlegt.

34 Ulrich Menzel, Konkurrierende Weltordnungsmodelle in historischer Perspektive, in; KAS/Auslandsinformationen, 20. Jg., Heft 6, (2004), S. 4-25 (23). 
jeden Kompromiss aus. Weil die Geschichte uns lehre, dass Großmächte auf die Dauer nicht vernünftig bleiben, wird jedes noch so kleine Anzeichen in der US Politik als unumstößliches Zeichen dafür gewertet, dass dieser Absturz nun beginnt. Andere Autoren sind dann auch mit Thesen zur Hand, derzufolge der Abstieg der USA als Großmacht bereits einsetze oder unvermeidlich sei. ${ }^{35}$

Eng verbunden hiermit ist die dritte falsche Prämisse. Sie lautet: Es gibt einen grundsätzlichen ordnungspolitische Dualismus zwischen den USA und Europa: den zwischen hegemonialem Unilateralismus und Multilateralismus. ${ }^{36}$ Diese Prämisse ist zum einen falsch, weil sie ein geistiges Eigentum reklamiert, welches nicht den Europäern zusteht. Es waren vor allem die USA, die nach dem Zweiten Weltkrieg die auch bis heute bestehenden Formen des Multilateralismus geschaffen oder direkt bzw. indirekt initiiert haben - zumeist gegen den Widerstand gerade jener Staaten Europas, die sich heute als Verteidiger des Multilateralismus geben. Sie ist auch deshalb falsch, weil sie suggeriert, dass die USA nicht mehr im Multilateralismus mitwirken wollen oder würden. Vielmehr ist festzuhalten, dass es bestimmte Formen und Bereiche des Multilateralismus gibt, die in den USA (und zwar nicht nur bei Neokonservativen und Republikanern) mit zunehmender Kritik gesehen werden, während in anderen Bereichen die Mitwirkung der USA in multilateralen Verhandlungsforen oder Organisationen kein prinzipielles Problem zu sein scheint. Diese Prämisse ist zudem schädlich, weil sie Multilateralismus wiederum nur auf prozedurale Regeln reduziert, nicht jedoch auf die Substanz, d.h. die mit Hilfe des Multilateralismus $\mathrm{zu}$ lösenden Probleme. ${ }^{37}$ Multilateralismus ist während der vergangene 60 Jahre in keinem Fall geschaffen worden, um die USA oder eine andere Macht einzubinden, sondern multilaterale Organisationen (wie Vereinte Nationen, ihre vielen Unterorganisationen, die EU etc.) und Verhandlungsgremien sind geschaffen worden, um internationale Probleme zu lösen, die mit den bis dahin bestehenden Instrumenten nicht zu lösen waren. Wenn mittlere und kleinere Staaten heute einen positiven Nebeneffekt von Multilateralismus darin sehen, dass sie bei der Regelung internationaler Politik mitreden und mitwirken können, so ist das ein kluger und durchaus beabsichtigter Nebeneffekt, aber doch niemals der Hauptzweck von multilateralen Organisationen oder Verhandlungsgremien gewesen. Hier vermittelt die Theorie ein verzerrtes Bild der Realität, indem sie einen eher sekundären Aspekt des Multilateralismus herausgreift.

Das Problem mit dem Multilateralismus im transatlantischen Verhältnis nimmt aber genau an diesem Dualismus

35 Charles A. Kupchan, The End of the American Era. U.S. Foreign Policy and the Geopolitics of the Twenty-First Century. New York 2002.

36 Vgl. Menzel, a.a.O., S. 23; siehe Thomas Risse, Die neue Weltordnung. US amerikanische Hypermacht - europäische Ohnmacht?, in: WeltTrends, Nr. 39, 2003, S. 110-119.

37 Typisch für diese Verengung ist das folgende Zitat: »Multilateralismus verlangt nun einmal, dass man bereit ist, sich den einvernehmlichen gesetzten Regeln auch zu unterwerfen. Die Unterwerfung möglichst vieler anderer unter selbstgesetzte Regeln zu verlangen ist hingegen kein Multilateralismus, eben diesen Stil werfen jedoch amerikanische Kritiker nicht zu Unrecht der eigenen Regierung vor «, Harald Müller, Das transatlantische Risiko. Deutungen des amerikanisch-europäischen Weltordnungskonflikts, in: Aus Politik und Zeitgeschichte, B 3-4 (2004), S. 7-17 (S. 7). von Prozedur und Substanz seinen Ausgang: Während für die USA Multilateralismus in der Regel die Aufgabe hat, Probleme zu lösen, stehen für Europäer die prozeduralen Aspekte im Vordergrund. ${ }^{38}$ Dies geht so lange gut, wie multilaterale Institutionen beiden Zwecken gerecht werden. In dem Augenblick, wo die substanzielle Zwecksetzung bei multilateralen Institutionen fragwürdig wird, neigen die USA erst dazu, die Reform des Multilateralismus zu probieren. Sofern das nicht oder nicht ausreichend gelingt, setzen dann meistens unilaterale Maßnahmen ein. Dem geht oft eine Phase voraus, in dem amerikanischer Reformwunsch und europäischer Strukturkonservativismus gegeneinander stehen. Dies lässt sich anhand von Entwicklungen im Bereich der Nichtverbreitungspolitik ebenso aufzeigen wie im Bereich der kollektiven Sicherheit. ${ }^{39}$

Im Prinzip ist es ja normal und nachvollziehbar, wenn kleinere und mittlere Staaten großen Wert darauf legen, dass ihre Stimme gehört wird und ihre Interessen und Sichtweisen von den großen Mächten in dauerhafter und verbindlicher Weise berücksichtigt werden, aber diese Erwartung geht nur auf, wenn die Vertreter dieser Staaten auch dafür sensibel bleiben, dass Multilateralismus effektiv bleibt. Ansonsten riskiert man, dass das Interesse an multilateraler Kooperation bei derjenigen Macht nachlässt, die letztendlich die größte internationale Verantwortung trägt (und deren Kooperation man auch sucht). Ein Großteil der heutigen Probleme im transatlantischen Bereich lässt sich darauf zurückführen, dass die Alliierten es nicht geschafft haben, die notwendige Balance zwischen der substanziellen und der prozeduralen Dimension des Multilateralismus zu wahren. Es fehlt in Europa weitgehend an einer Sensibilisierung dafür, dass Multilateralismus angepasst und gegebenenfalls auch radikal modifiziert werden muss, bzw. auch mal aufgegeben werden muss. Zwar hat die Formel vom »effektiven Multilateralismus « Eingang in die Kommuniquésprache der deutschen und europäischen Diplomatie gefunden, eine substanzielle Änderung der Politik hat sich aber noch nicht abgezeichnet. Was der Bush-Administration fehlt ist die Sensibilisierung dafür, dass man den Europäern dabei etwas helfen muss. Die Theoriedebatte in der Wissenschaft von den Internationalen Beziehungen und die damit einhergehende Verengung der Theorie der hegemonialen Stabilität haben dazu beigetragen, dass dieser Mangel an Sensibilität auf beiden Seiten bestehen bleibt, denn sie lenkt die Aufmerksamkeit nur auf einen nebensächlichen Teilaspekt.

Dass die Prämisse des ordnungspolitischen Dualismus falsch ist, sieht man auch dann, wenn man vergleicht, was als langfristige ordnungspolitische Ziele und Herausforderungen auf beiden Seiten des Atlantiks genannt wird. Sowohl amerikanische wie europäische Strategiedokumente

38 Vgl. Stanley Hoffmann, The United States and International Organizations, in: Robert J. Lieber (Hrsg.), Eagle Rules? Foreign Policy and American Primacy in the Twenty-First Century. Upper Saddle River, N.J. 2002, S. 342-352; Joachim Krause, Multilateralism - Behind European Views, in: The Washington Quarterly, Vol. 27, Spring 2004, Nr. 2, S. 43-59.

39 Details siehe in Joachim Krause, Multilateral Co-operation in the Face of Old and New Security Challenges, in: William Wallace and Young Soogil (Hrsg.), Asia and Europe. Global Governance as a Challenge to Cooperation. Tokyo, CAEC 2004, S. 58-91 (www.isuk.org). 
lassen im Wesentlichen ein an liberalen Werten orientiertes Friedens- und Ordnungskonzept erkennen und zeigen - etwa wenn man die Nationale Sicherheitsstrategie der USA vom September 2002 und das im Dezember 2003 von der EU verabschiedete Strategisch Konzept miteinander vergleicht weitgehende Übereinstimmung in der Frage der Risiken und Probleme sowie der einzuschlagende Maßnahmen. ${ }^{40}$

\section{Wie gefährdet ist die transatlantische Allianz?}

Wie oben gezeigt wurde, gibt es keinen Anlass für jene schicksalsschweren Verdikte, denen zufolge sich die USA jetzt ähnlich wie weiland die Athener nach dem Sieg gegen die Perser aufführen. Diese hatten erst die gemeinsame Kriegskasse geplündert, dann die früheren Alliierten unterworfen, um sich dann in einem endlosen Krieg mit den Spartanern zu verschleißen. Es gibt tatsächlich eine zunehmende Entfremdung innerhalb der westlichen Allianz, die in der Hauptsache (aber nicht ausschließlich) auf unterschiedlichen Vorstellungen über die Nützlichkeit multilateraler Institutionen bei der Lösung gemeinsamer Probleme beruht sowie auf Differenzen über den Einsatz militärischer Machtmittel zur Lösung internationaler ordnungspolitischer Aufgaben. Diese Divergenzen sind eigentlich keine Überraschung, denn sie reflektieren zum einen die offenkundige Krise des globalen Multilateralismus (insbesondere im Bereich der internationalen Sicherheit) und die Unterschiede in der Ernsthaftigkeit, mit der auf beiden Seiten des Atlantiks nach dem Ende des Ost-West-Konflikts die Auseinandersetzung mit den neuen Sicherheitsproblemen geführt und entsprechende institutionelle und kapazitätsmäßige Vorbereitungen getroffen worden sind. Das Aufkommen der (neo) konservativen Republikaner war nicht - wie so oft behauptet - die Ursache dieser Entwicklung. Sie ist aber durch diese Entfremdung gestärkt worden und hat entscheidend dazu beigetragen, dass diese Krise sich verschärfte. Diese Divergenzen bestehen aber auch so und werden nur dann zu lösen sein, wenn westliche Politiker und sonstige Meinungsführer (besonders in den Medien) auf beiden Seiten des Atlantiks darauf verzichten, durch unnötige Ideologisierung und Emotionalisierung eine letztlich sinnlose und selbstzerstörerische Debatte herbeizuführen bzw. fortzusetzen.

Worin liegt der Kern der Krise des Multilateralismus? Es wäre falsch von einer generellen Krise des Multilateralismus zu sprechen, denn es gibt so viele verschiedene Varianten davon mit unterschiedlicher Effektivität. Es empfiehlt sich daher nach unterschiedlichen Formen und Arten des Multilateralismus zu unterscheiden. Im Prinzip kann man mindestens zwei Formen des Multilateralismus unterscheiden: multilaterale Verhandlungsforen und multilaterale Organisationen. Daneben lassen sich die folgenden Arten des Multilateralismus unterscheiden: ${ }^{41}$

40 Siehe Joachim Krause, Multilaterale Ordnung oder Hegemonie? Zur transatlantischen Debatte über die weltpolitische Neuordnung, in: Aus Politik und Zeitgeschichte, B 31-32, 2003, S. 6-14.

41 Vgl. zum Folgenden Joachim Krause, Multilateral Co-operation in the Face of Old and New Security Challenges, a.a.O., S. 60-62.
1. Das System der Kollektiven Sicherheit unter der Charter der Vereinten Nationen: Dieses ist von der internationalen Staatengemeinschaft erklärtermaßen als internationale Sicherheitsordnung anerkannt. Im Zentrum dieser Ordnung steht der Sicherheitsrat der Vereinten Nationen.

2. Ökonomischer Multilateralismus: Dies ist ein System zur Sicherung bzw. Förderung von Freihandel und internationalem Zahlungsverkehr sowie zur Lösung einer Vielzahl von Problemen, die im Rahmen der Liberalisierung des Handels und der Ausweitung der internationalen Arbeitsteilung entstanden sind. Insbesondere soll die internationale Entwicklung gefördert werden. Ursprünglich sollten vor allem der Wirtschaftsrat der Vereinten Nationen (ECOSOC) und die Welthandelskonferenz diese Funktion erfüllen. Tatsächlich haben die Sonderorganisationen Weltbank und Währungsfonds und regionale Entwicklungsbanken sowie die Verhandlungsrunde GATT dazu geführt, dass heute Freihandel und internationale Arbeitsteilung eine Realität geworden sind.

3. Offener funktionaler Multilateralismus: Hier handelt es sich um Verhandlungsforen und internationale Organisationen, die die Aufgabe haben, bestimmte Probleme aufzugreifen und gemeinsam Lösungen zu entwickeln. Die Zahl der Themen ist im Prinzip unendlich, es kann sich um Fragen der Umweltpolitik handeln (Klima) aber auch um Sicherheitsfragen (multilaterale Abrüstung) oder um Themen wie Artenschutz. Die Mitwirkung steht im Prinzip allen Staaten offen (bzw. wenn es sich um einen regional begrenzten offenen Multilateralismus handelt, steht er allen Staaten der Region offen). Sie kann auch auf Nichtregierungsorganisationen ausgedehnt werden (global governance). Hauptakteur des offenen Multilateralismus sind die Vereinten Nationen und ihre Sonderorganisationen. In fast allen Bereichen des offenen funktionalen Multilateralismus herrschen ähnliche Verfahren der Entscheidungsbildung und Organisation.

4. Geschlossener funktionaler Multilateralismus: Hierbei handelt es sich um Verhandlungsforen oder permanente Einrichtungen, die bestimmte Probleme (oder Bündel von Problemen) lösen sollen, wo aber Wert darauf gelegt werden soll, dass nur wenige Staaten daran teilhaben. Die Gipfeltreffen der G8, die verschiedenen Exportkontrollregime aber auch eine überregionale Sicherheitsorganisation wie die NATO gehören hierzu.

5. Gemeinschaftlicher Multilateralism: Hierbei handelt es sich um eine Form der breiten und kontinuierlichen Form der Zusammenarbeit zwischen gleichgerichteten Staaten, die eine solche Verstetigung und Tiefe erfährt, dass die Grenze zur Vergemeinschaftung fließend werden kann. Bislang gibt es nur ein Beispiel dafür: Das ist die Europäische Union, wenngleich ASEAN und MERCOSUR zumindest darum bemüht sind, dem Beispiel der Europäer teilweise zu folgen.

Schaut man sich die amerikanische Debatte zum Multilateralismus an, so wird sehr schnell erkennbar, dass in den vergangenen 15 Jahren vor allem zwei Formen in das Zentrum der Kritik geraten sind: das System der Kollektiven Sicherheit sowie der offene funktionale Multilateralismus. 
Kollektive Sicherheit besteht nicht nur aus dem Gewaltverbot der Charter der Vereinten Nationen, sondern es ist ein anspruchsvolles und komplexes System der Übertragung der Verantwortung für die Sicherheit (regional wie global) auf den Sicherheitsrat. ${ }^{42}$ Dieser soll sowohl (im Zusammenwirken mit anderen Organen der VN) zwischenstaatliche Konflikte gewaltlos helfen lösen (Kapitel VI) als auch international die Ordnung gegenüber Staaten oder anderen Akteuren aufrechterhalten, die den Frieden und die internationale Sicherheit bedrohen oder stören (Kapitel VII). Dem Sicherheitsrat ist hierzu die primäre Verantwortung für den Weltfrieden übertragen worden. Während der Zeit des Ost-West-Konflikts war dieses System weitgehend inaktiv, nach dessen Ende hatten aber 1992 die Mitgliedstaaten des Sicherheitsrates auf einem Gipfeltreffen die Gültigkeit dieses Systems unterstrichen und entsprechende Unterstützung gefordert.

Heute ist die Bilanz von Kapitel VI durchaus so, dass von einem Nebeneinander von positiven und negativen Erfahrungen gesprochen werden kann, die sich weiter entwickeln lassen. ${ }^{43}$ Was Kapitel VII betrifft, so ist die Bilanz weitgehend negativ. Nicht einen einzigen großen Konflikt der 90er Jahre hat der Sicherheitsrat lösen können und keiner derjenigen Akteure, die die internationale Ordnung herausgefordert hatten, wurde durch Einschreiten der Vereinten Nationen in die Schranken gewiesen. Ursache war in der Regel die Uneinigkeit unter den ständigen Mitgliedern. Vor allem angesichts der Vertreibungskriege und Genozide im früheren Jugoslawien und in Zentralafrika hat der Sicherheitsrat seine satzungsmäßigen Aufgaben nicht erfüllt. Und auch das anfängliche Paradebeispiel Kollektiver Sicherheit - die Reaktion auf die Besetzung Kuwaits durch den Irak - hat in den 90er Jahren seinen Glanz verloren. Diese Entwicklungen sind ausgiebig in der akademischen Literatur kritisiert worden. ${ }^{44}$ Sie lassen nur den einen Schluss zu: Kollektive Sicherheit als internationale Ordnung hat versagt. Diese Erkenntnis wird auch in der politischen Debatte in den USA reflektiert. Sie hat zu politischen Entscheidungen geführt, die weitgehende Konsequenzen für das System der Kollektiven Sicherheit hatten und die in Europa zumeist kaum registriert worden sind: Im Jahr 1995 etwa verabschiedete der Kongress eine Gesetzgebung, die das Verhalten des Sicherheitsrates im Bosnien-HerzegowinaKonflikt als Bruch des Völkerrechts qualifizierte und unilaterale Schritte der USA forderte, um die Ziele der Charta der Vereinten Nationen durchzusetzen. Drei Jahre später verabschiedete dasselbe Gremium ein Gesetz, welches feststellte, dass es keinen Sinn mehr mache, den Irak mit einem Rüstungskon-

42 Mohammed Ayoob, Squaring the Circle: Collective Security in a System of States, in: Thomas G. Weiss (Hrsg.), Collective Security in a Changing World, Boulder, Col. 1993, S. 45-62, sowie Leon Gordenker and Thomas G. Weiss, The Collective Security Idea and Changing World Politics, ebenda, S. 3-18.

43 Lakhdar Brahimi (Chairman), Report of the Panel on United Nations Peace Operations, United Nations Document A/55/303 bzw. S/2000/809, New York, 20. August 2000.

44 Vgl. Steven L. Burg and Paul K. Shoup, The War in Bosnia-Herzegovina: Ethnic Conflict and International Intervention, London 1999; Tim Judah, Kosovo: War and Revenge, London and New Haven 2000; Alison L. Des Forges, Leave None to Tell the Story: Genocide in Rwanda Washington, D.C. (Human Rights Watch) 1999; Tim Trevan, Saddam's Secrets: The Hunt for Iraq's Hidden Weapons, London 1999. Samantha Power, A Problem from Hell. America and the Age of Genocide. New York 2002; Rosemary Righter, Utopia Lost: The United Nations and World Order, New York 1995. trollregime zu überziehen, weil das Regime Saddam Husseins niemals jene Transparenz zulassen werde, die nötig sei um das Rüstungskontrollregime von 1991 zu überprüfen. Vier Jahre später stellte der US Kongress fest, dass der Sicherheitsrat im Falle des Iraks seine Aufgaben nicht erfüllt habe und dass daher die USA einseitige militärische Maßnahmen vornehmen müssten. ${ }^{45}$ Dies sind nicht - wie in der politikwissenschaftlichen Analyse zu vernehmen - eigene Regeln, die von den USA neu aufgestellt wurden, sondern die Erinnerung daran, dass der Sicherheitsrat nicht in der Lage war (und ist), seinen satzungsmäßigen Aufgaben und den dort gesetzten Regeln gerecht $\mathrm{zu}$ werden. Dies ist der Kern des amerikanischen Unilateralismus und nicht die immer wieder behauptete grundsätzliche Neigung zum Multilateralismus a la carte. Was man aber sehen muss, ist, dass es sehr unterschiedliche Formen der Kritik an der Kollektiven Sicherheit in den USA gibt: Diese reicht von moderaten Stimmen, die inkrementelle Reformen der Vereinten Nationen für ausreichend halten, hin zu jenen, die größere Reformen des Multilateralismus für notwendig halten bis hin zu jenen Neokonservativen, die Multilateralismus für prinzipiell schädlich halten. ${ }^{46}$

Der offene und funktionale Multilateralismus wird aus etwas anderen Gründen kritisiert. Hier wird in erster Linie darauf verwiesen, dass multilaterale Institutionen nicht nur schwerfällig seien - womit manche leben können - aber dass diese dazu tendierten, sich in Eigenbeschäftigung und Ideologisierung zu verlieren. Die Zunahme anti-israelischer und antiwestlicher (insbesondere anti-amerikanischer) Tendenzen in den Vereinten Nationen, die Unergiebigkeit der meisten UN Unterorganisationen sowie die Gefahr der Verselbständigung von Themen bis hin zu sinnlosen Regulierungen werden dabei in den USA kritisiert. Auch hier ist ein breites Spektrum zu erkennen. Auf der einen Seite findet man jene, die einzelne Aspekte des offenen und funktionalen Multilateralismus kritisieren, aber eine weitgehend positive Einstellung zum offenen Multilateralismus behalten, auf der anderen Seite diejenigen, die die Vereinten Nationen abschaffen oder in ihren Funktionen auf eine Redeorganisationen beschränken wollen. Erkennbar ist aber ein zunehmendes Misstrauen in die Problemlösungskapazität der Vereinten Nationen und anderer offener multilateraler Institutionen. Auch die Ablehnung des Kyoto-Protokolls durch die Bush-Administration findet in den USA breitere Unterstützung als hier gemeinhin vermutet wird. Ursache ist die Furcht, dass ein durchaus bestehendes Problem ideologisch überhöht und dramatisiert wird und in der Folge überflüssi-

45 Der Bosnia Herzegovina Self Defense Act wurde am 26. Juli 1995 vom Kongress verabschiedet. Er trat nie in Kraft, weil Präsident Clinton sein Veto einlegte. Er führte aber zu einer radikalen Kurswende der amerikanischen Politik gegenüber Bosnien-Herzegowina. Der Iraq Liberation Act wurde am 31. Oktober 1998 verabschiedet (Public Law 105-338). Am 11. Oktober 2002 verabschiedete der US Kongress eine Resolution, in der er den amerikanischen Präsidenten zum Einsatz von Gewalt autorisierte, um alle relevanten Resolutionen der Vereinten Nationen zu erzwingen.

46 Vgl die drei sehr unterschiedlichen Formen der Kritik vgl. John G. Ruggie, This Crisis of Multilateralism Is Different, Speech delivered at the UNA-USA National Forum on the United Nations, June 26, 2003; www.unusa.org; John Van Oudenaaren, What is 'Multilateral'?, in: Policy Review, Nr 117, Februar 2003, S. 33-47; Charles Krauthammer, The Unipolar Moment Revisited, in: The National Interest, Nr. 70 (Winter 2002/2003), S. 5-17. 
ge Regulierungen und Wettbewerbsnachteile für die amerikanische Wirtschaft entstehen können.

Kritik am geschlossenen funktionalen Multilateralismus ist weniger stark ausgeprägt, aber es nimmt - nicht nur unter neokonservativen Autoren - die Kritik an der NATO zu, weil diese zu unflexibel sei und weil vor allem von den Franzosen angenommen wird, sie würden multilaterale Institutionen in der Hauptsache dazu nutzen, um die USA zu bremsen. Dennoch gilt die NATO als deutlich effizientere Form des Multilateralismus als etwa die Vereinten Nationen.

Ein Kernpunkt der Debatte über Kollektive Sicherheit ist die Rolle militärischer Gewalt. Zwar betonen die meisten Politiker in Europa und den USA, dass natürlich gesichert sein müsse, dass militärische Gewalt zur Erzwingung internationaler Ordnung eingesetzt werden kann. Aber die Bedingungen, die erfüllt sein müssen, bis es dann zum Einsatz der »ultima ratio « kommen darf, sind bei europäischen Regierungen so eng definiert, dass eigentlich kaum ein zeitgerechter militärischer Einsatz unter Kapitel VII der VN Charter möglich erscheint. Anders in den USA: Dort wird die Rolle militärischer Gewalt zur Erzwingung internationaler Ordnung und der Wiederherstellung von Frieden ganz anders angegangen als in den meisten Ländern Europas. Was viele Europäer übersehen ist, dass die von ihnen geltend gemachten humanitären Überlegungen (Anwendung von Gewalt führt zu Opfern) eher für als gegen militärische Interventionen sprechen: Die Zahl der Todesopfer internationaler, von den USA angeführten Interventionen während der vergangenen 15 Jahre lag unter 50000 Menschen (Soldaten und Zivilisten), die Zahl der Opfer von versäumten Interventionen der »Völkergemeinschaft « dürfte im gleichen Zeitraum bei über 5 Millionen Toten gelegen haben.

Die Ursachen für die Unterschiede beim Umgang mit militärischer Gewalt sind differenziert zu sehen. Zum einen wirken hier historische Erfahrungen nach, insbesondere der II. Weltkrieg. Aber es wird auch erkennbar, dass die jeweiligen Reformen der militärischen Apparate nach dem Ende des Ost-West-Konflikts unterschiedlich konsequent durchgeführt worden sind: in den USA ist mit relativ großer Klarheit und Konsequenz eine Reform durchgezogen worden, die es vor allem erlauben soll, weltweit militärisch zu operieren - und zwar mit einer hohen Wahrscheinlichkeit des Erfolgs und einer ebenso hohen Wahrscheinlichkeit geringer Eigenverluste und humanitärer Kollateralschäden. In Europa sind die entsprechenden nationalen Militärreformen nur schleppend vorangekommen, insbesondere die Bundesrepublik Deutschland hat sich sehr viel Zeit genommen. ${ }^{47}$ Zudem ist die Vielfalt der nationalen Streitkräfte in Europa ein Hindernis für eine Modernisierung, die sich an den Gegebenheiten der heutigen Sicherheitspolitik orientiert. Zusammen genommen geben die EU-Staaten sehr viel Geld für Verteidigung aus (etwa die Hälfte des Betrags der USA). Sie haben mehr Soldaten und mehr Kampfflugzeuge und Panzer als

47 Vgl. Roland Kaestner, Streitkräftereform und internationaler Wandel, in: S und F: Vierteljahresschrift für Sicherheit und Frieden, Jg. 19, Nr. 2, 2001, S. 56-61; Bernhard Fleckenstein, Bedingt einsatzfähig: Der lange Weg zur Neugestaltung der Bundeswehr, in: Aus Politik und Zeitgeschichte, B 43,2000, S. 13-23. die USA. Aber sie verfügen nur über einen Bruchteil jener militärischen Kapazitäten, die unter den heutigen strategischen Bedingungen notwendig sind. ${ }^{48}$ Hätte die EU 1990 im Bereich der Verteidigungspolitik den Weg der Vergemeinschaftung wie bei der Währungspolitik eingeschlagen, dann stünde Europa heute anders da. Dann würde mehr Geld in neue Strukturen und intelligentere Waffensysteme investiert worden sein. Stattdessen wurde viel Geld für die Erhaltung alter Strukturen und Waffensysteme ausgegeben. Zwar haben wir eine Vielzahl von Bemühungen, europäische Verteidigungspolitik zu koordinieren, und es sind hierbei auch beachtliche Erfolge erreicht worden, aber der Preis war hoch: In den 90er Jahren und auch danach ist die Hauptaufmerksamkeit der europäischen Außen- und Verteidigungspolitik durch die Beschäftigung mit innereuropäischen institutionellen und prozeduralen Problemen absorbiert gewesen. Die Folge war, dass die notwendige Auseinandersetzung mit den eigentlichen außenpolitischen Problemfällen hintan gestellt wurde (Beispiel die Kriege im früheren Jugoslawien). Auch weiterhin behindern nationale Egoismen, Mangel an Geld und Uneinigkeit über die Zielrichtung der europäischen Politik eine effiziente Nutzung der vorhandenen Ressourcen für das Ziel, eine militärische Kapazität zur Verfügung zu haben, die den Europäern außenpolitische Handlungsfähigkeit erlaubt.

Die Auseinanderentwicklung bei der militärischen Reform ist seit Beginn der 90er Jahre beobachtet und wegen der zu erwartenden Konsequenzen immer wieder kritisiert worden. ${ }^{49}$ Geholfen hat das nichts, denn die Fähigkeitslücke im Bereich der Streitkräfte und der Rüstungstechnologien hat sich weiter geöffnet. Für die US-Streitkräfte sind die europäischen Partner immer weniger interessant, weil sie nur in Teilbereichen kompatibel sind. Zudem sind Einsatzgrundsätze und politische Vorgaben oft schwer zu harmonisieren. Diese Fähigkeitslücke trägt natürlich zur politischen Auseinanderentwicklung bei, da die USA heute über militärische Optionen in ihrer Außenpolitik verfügen, die die Europäer nicht haben. Das führt in der Tendenz dazu, dass in den USA die Lösbarkeit von Problemen mit Hilfe militärischer Mittel und in Europa die Effizienz von multilateralen Institutionen überschätzt werden. Dabei können sich Mythen und gegenseitige Schuldzuweisungen entwickeln, die dann zu Unilateralismus beitragen, bzw. zu neuen Formen des Multilateralismus führen, die die USA ausgrenzen (wie etwa bei dem Ottawa-Abkommen zum Verbot von Landminen).

Davon unberührt bleibt die Frage, wer sichert die internationale Ordnung gegen Akteure, die ihre Regeln bewusst herausfordern oder die Bedrohungen der internationalen oder regionalen Sicherheit verursachen, insbesondere solche, die Genozid, ethnische Vertreibung oder massive Verwüstungen

48 Varwick, Die Zukunft der transatlantischen Sicherheitsbeziehungen, a.a.O., S. 142 .

49 Lothar Rühl, Die Bundeswehr-Reform aus bündnispolitischer Sicht, in: Aus Politik und Zeitgeschichte, B 43, 2000, S. 3-11; David Yost, The NATO Capabilities Gap and the European Union, in: Survival, Vol. 42, Nr. 4, Winter 2000-2001, S. 97-128; Uwe Nerlich, Strategische Dimensionen der Informationskriegführung, in: Europäische Sicherheit, Jg. 47, Heft 4, April 1998, S. 40-43; Christian Tuschhoff, Die politischen Folgen der Streitkräfte-Reform der NATO, in: Aus Politik und Zeitgeschichte, B15/16, 1993, S. 28-39. 
vornehmen? Der Sicherheitsrat der Vereinten Nationen wird dieser Aufgabe in absehbarer Zeit nicht nachkommen können. Hiergegen spricht die Struktur mit dem Veto-Recht der ständigen Mitglieder. Und selbst wenn es $\mathrm{zu}$ einer Reform käme, bei der das Veto-Recht abgeschafft oder eingeschränkt würde, bleibt große Skepsis bestehen. Das Hauptproblem ist der Mangel an Einheitlichkeit und oft kommt die Neigung vieler Akteure hinzu, Multilateralismus als Instrument zu nutzen, um Verantwortlichkeit für Nichtstun abzuwälzen. Auch eine Erweiterung des Sicherheitsrates um neue Mitglieder wird daran nichts ändern, es dürfte eher die Strukturprobleme verstärken. ${ }^{50}$ Die einzige wirklich vielversprechende Strategie dürfte die Verlagerung der Aufgabe der Ordnungswahrung auf eine Institution des geschlossenen Multilateralismus sein. Da käme eigentlich nur die NATO in Frage. Sie ist die einzige Organisation, welche zugleich militärische Handlungsfähigkeit und ein hohes Maß an Legitimität für internationale Interventionen herstellen kann. Dies hat sich am Fall der Intervention im Kosovo gezeigt und es wäre ein guter Neustart für die NATO, an den Erfahrungen von Kosovo anzuknüpfen. ${ }^{51}$ Das würde bedeuten, dass erst einmal innerhalb der Atlantischen Allianz eine gemeinsame Bestimmung der Ziele und eine Bewertung der Stärken und Schwächen vorgenommen wird. Dann könnte sich die NATO auch offen und völkerrechtlich verbindlich als eine Institution $\mathrm{zu}$ verstehen geben, die angesichts schwerwiegender Bedrohungen der internationalen Sicherheit oder angesichts schwerer humanitärer Katastrophen sich das Recht vorbehält einzugreifen, wenn der Sicherheitsrat der Vereinten Nationen zu keinen effektiven Handlungen fähig ist.

Dass sich die NATO schon stückweise in diese Richtung bewegt, ist deutlich erkennbar. Die noch bis 1999 bei Deutschen und vor allem Franzosen bestehenden Vorbehalte gegen die Befassung der NATO mit Krisen außerhalb des nordatlantischen Raumes sind mittlerweile aufgegeben worden. Dadurch ist der Weg frei, die NATO zu einem multilateralen Konsultationsrahmen für den Umgang mit Krisen in praktisch jedem Winkel der Erde zu machen. Hätte dieser Konsultationsrahmen schon 2002 bestanden, dann hätte die große diplomatische Krise über den Irak wahrscheinlich vermieden (oder aber in ruhigere Gewässer verlagert) werden können.

Ein ganz zentrales Element wird aber die NATO Response Force (NRF) sein, eine Interventionstruppe von 21000 Mann, die nach kurzer Vorbereitungszeit weltweit verlegt werden kann. Ihre Einrichtung wurde auf dem Prager Gipfel der NATO beschlossen und sie soll spätestens 2006 einsatzbereit sein. Zusammen mit den weiterhin bestehenden amerikanischen Kräften sowie der zu schaffenden europäischen Interventionstruppe würde dann ein erhebliches Interventionspotential bestehen, welches der NATO die Möglichkeit verschaffen würde, internationale Ordnungsaufgaben wahrzunehmen, sofern diplomatische Mittel nicht hinreichen.

50 Thomas Weiss, The Illusion of UN Security Council Reform, in: The Washington Quarterly, Vol. 26, Nr. 4, Autumn 2003, S.147-161.

51 Vgl. Joachim Krause, Der Kosovo-Krieg - ein Schlüsselereignis westlicher Sicherheitspolitik, in: Die Internationale Politik 1999/2000 - Jahrbuch der Deutschen Gesellschaft für Auswärtige Politik, hrsg. von Wolfgang Wagner, Marion Gräfin Dönhoff, Lutz Hoffmann, Karl Kaiser, Werner Link und Hanns Maull, München 2002, S. 125-136.

\section{Perspektiven der NATO}

Wie diese Überlegungen erkennen lassen, sehen die Perspektiven für die nordatlantische Allianz gar nicht so schlecht aus. Das große Plus der NATO ist, dass es sich bei ihr um eine relativ effiziente multilaterale Institution handelt. Sie ließe sich in eine Richtung weiter entwickeln, in der Amerikaner wie Europäer gemeinsam an der Lösung konkreter Sicherheitsprobleme arbeiten und wo sie auch das nötige diplomatische und militärische Instrumentarium besitzen, um handlungsfähig zu sein. Dies alles setzt aber voraus, dass die mit so großer Emotionalität belastete Krise über den Irak nicht nur überwunden wird, sondern auch in eine strategische Neubewertung dessen eingeht, was die Staaten der nordatlantischen Gemeinschaft an gemeinsamen Problemen vor sich haben und wie sie diese am Besten lösen können. In diesem Zusammenhang wäre auch eine Bewertung der Leistungsfähigkeit der verschiedenen Arten von Multilateralismus sinnvoll. Nur so kann gesichert werden, dass Multilateralismus einerseits der Bewältigung sachlicher Probleme dient, andererseits aber auch eine Form der Mitbestimmung und Mitwirkung von kleinen und mittleren Staaten (und somit der Einbindung der USA) wird. Der Streit über die Rolle von Multilateralismus und von Gewalt zur Aufrechterhaltung internationaler Ordnung könnte auch im Rahmen einer Organisation wie der NATO gelöst oder zumindest pragmatisch überwunden werden. Die Antwort auf die Krise des globalen Multilateralismus liegt weder in der Ablehnung von Multilateralismus noch in der Ignorierung von deren Schwächen, sondern in der stärkeren Nutzung effektiver Formen des Multilateralismus - und da gehört die NATO eigentlich zu den effektivsten Organisationen und sollte der Kern einer neuen transatlantischen Gemeinsamkeit sein. Das bedeutet, dass der Westen neu erfunden werden muss - und zwar mit Blick auf neue gemeinsame Herausforderungen. ${ }^{52}$

Das ist leichter gesagt als getan, denn die Beseitigung des politischen Schadens der Irak-Krise wird noch lange andauern. Sie erfordert von amerikanischer Seite, dass man sich stärker mit den Bedenken und Anliegen der europäischen Partner auseinandersetzt und diese nicht öffentlich herabwürdigt und lächerlich macht. ${ }^{53}$ Sie erfordert auf europäischer Seite ein stärkeres Bewusstsein dafür, dass es außerordentlich schwierige Problemlagen gibt, die nicht nur mit Mitteln der Diplomatie gelöst werden können und dass man eine enge Kooperation mit den USA braucht, um dieser Probleme Herr zu werden. ${ }^{54}$ Es bedarf auch auf europäischer Seite der Prüfung, ob die eigenen außen- und sicherheitspolitischen Instrumente angesichts der Herausforderung durch einen islamistischen Terrorismus, der Proliferation von Massenvernichtungswaffen und der sich abzeichnenden Probleme der Energiesicherheit auf die Dauer ausreichend

52 Dominique Moisi, Die Wiedererfindung des Westens, in: Internationale Politik, Jg. 58, Heft 12, Dezember 2003, S. 21-44; s. a. Francois Heisbourg, US-European relations: from lapsed alliance to new partnership? in: International Politics (The Hague), Vol. 41, Nr. 1, März 2004, S. 119-126.

53 Vgl. Ivo H. Daalder, The End of Atlanticism, in: Survival, Vol. 45, Nr. 2 , Sommer 2003, S. 147-165.

54 Vgl. James Steinberg, An Elective Partnership. Salvaging Transatlantic Relations, in: Survival, Vol. 45, Nr. 2, Sommer 2003, S. 117-145. 
sind. Die neue Sicherheitsstrategie vom Dezember 2003 bietet erste Ansätze hierfür, bleibt aber noch recht vage

Dabei ist es auch wichtig, die Dynamik des sich gegenseitig verstärkenden Unilateralismus und Populismus zu durchbrechen. Wir befinden uns nicht an einem Scheitelpunkt der Geschichte, wo es darum geht, dass jetzt das Völkerrecht gegen das Recht des Stärkeren ins Feld geführt werden muss, oder wo es darum geht, die ungefesselte Hegemonie der USA einzuhegen. Vielmehr sind pragmatische Lösungen für den gemeinsamen Umgang mit dringlichen Problemen gefragt, nicht Rezepte aus dem 19. Jahrhundert.

\title{
Der Strategiewandel amerikanischer Außenpolitik und die Folgen für die transatlantischen Beziehungen
}

\author{
Peter Rudolf*
}

\begin{abstract}
Under the presidency of George W. Bush, changes in American grand strategy have, in effect, amounted to a rather consistent new paradigm of hegemonic unilateralism: the preservation of unipolarity, i.e., the maintenance of military supremacy regardless of potential threats and adversaries; a heightened perception of intolerable threats, which has led to the rejection of containment as the fundamental concept of security policy with respect to the new threats; the attempted legitimization of preventive war against states that support terrorism in whatever form, and the emphasis on strategic independence. Those changes, which culminated in the Iraq war, have accentuated structural problems in the transatlantic relationship.
\end{abstract}

Keywords: Strategiewandel, US-Außenpolitik, Irak-Intervention, transatlantische Kooperation, Naher und Mittlerer Osten

$\mathrm{T}$ Trotz der Wahrnehmung einer gemeinsamen Bedrohung durch den islamistischen Terrorismus hat der Strategie-wandel amerikanischer Außenpolitik nach dem 11. September die Konflikte im transatlantischen Verhältnis akzentuiert. ${ }^{1}$ Schärfer wurden die Konflikte vor allem deshalb, weil vor dem Hintergrund einer erhöhten Wahrnehmung asymmetrischer Bedrohungen die auf überlegene militärische Macht und uneingeschränkte Handlungsfreiheit setzende Denkschule den außenpolitischen Diskurs bestimmte und in beträchtlichem Maße die Außenpolitik unter George W. Bush prägte. Der Krieg gegen den Irak - geführt zur Ausschaltung einer sich angeblich mehr und mehr zuspitzenden künftigen Bedrohung - war Konsequenz und Kulmination der neuen strategischen Orientierung amerikanischer Außenpolitik, wie sie nach dem 11. September 2001 Gestalt gewann. ${ }^{2}$ Die traumatische Erfahrung asymmetrischer Verwundbarkeit veränderte zudem den Kontext außenpolitischer Entscheidungen tief-greifend: Sie führte zum erneuten Primat einer an »worst case «Annahmen ausgerichteten Sicherheitspolitik; damit verbunden zur klaren Dominanz eines seine historische Aufgabe findenden Präsidenten und $\mathrm{zu}$ einer selbstbeschränkten, ja bis zur institutionellen Selbstaufgabe gehenden Rolle des Kongresses in der Außenpolitik.

\footnotetext{
* Dr. Peter Rudolf, Forschungsgruppe Amerika, Stiftung Wissenschaft und Politik, Berlin.
}

\section{Der 11. September und seine Folgen: Ein neues strategisches Paradigma}

Die Anschläge des 11. September aktivierten das von Historikern diagnostizierte Streben nach $»$ absoluter Sicherheit «. ${ }^{3}$ In der Rhetorik des Präsidenten blitzt dieses Motiv seitdem immer wieder auf. Sollen die USA sich jemals wieder sicher fühlen können, muß die Bedrohung durch Terroristen und die unterstützenden Staaten in toto beseitigt werden. ${ }^{4}$ Der »Krieg gegen den Terrorismus« - und zwar in seiner staatszentrierten, gegen »Terrorstaaten « mit Massenvernichtungs-

1 Aus der Fülle der Arbeiten zu den transatlantischen Beziehungen siehe Helga Haftendorn, A Poisoned Relationship? Die transatlantischen Beziehungen nach den Terrorangriffen des 11. September 2001, in: Werner Kremp/Jürgen Wilzewski (Hrsg.), Weltmacht vor neuer Bedrohung. Die Bush-Administration und die US-Außenpolitik nach dem Angriff auf Amerika, Trier: Wissenschaftlicher Verlag Trier 2003, S. 249-275; Gert Krell, Arroganz der Macht, Arroganz der Ohnmacht. Die Weltordnungspolitik der USA und die transatlantischen Beziehungen, in: Aus Politik und Zeitgeschichte, B 31-32/2003 (28. Juli 2003), S. 23-30; Thomas Risse, Die neue Weltordnung: US-amerikanische Hypermacht - europäische Ohnmacht, in: WeltTrends, (Sommer 2003) 39, S. 110-119; Peter Rudolf, Die USA und die transatlantischen Beziehungen nach dem 11. September 2001, in: Aus Politik und Zeitgeschichte, B 25/2002 (21. Juni 2002), S. 7-13; John Peterson/Mark A. Pollack (Hrsg.), Europe, America, Bush: Transatlantic relations in the twenty-first century, London/New York: Routledge 2003; Gustav Lindstrom (Hrsg.). Shift or Rift? Assessing US-EU relations after Iraq, Paris: European Unions Institute for Security Studies 2003.

2 Zur Entwicklung der Außenpolitik unter Bush siehe insbesondere Ivo Daalder/James M. Lindsay, America Unbound: The Bush Revolution in Foreign Policy, Washington: Brookings Institution Press 2003.

3 James Chace/Caleb Carr, America Invulnerable. The Quest for Absolute Security from 1812 to Star Wars, New York: Summit Books 1988.

4 »We will not be secure as a nation until all of these threats are defeated. Across the world and across the years, we will fight these evil ones, and we will win. « George W. Bush, Rede in Fort Campbell, 21.11.2001, http://www.whitehouse.gov/news/releases/2001/11/print/200111213.html. 